Check for updates

Cite this: RSC Adv., 2019, 9, 21989

\title{
Surface protolytic property characterization of hydroxyapatite and titanium dioxide nanoparticles $\uparrow$
}

\author{
Ekaterina Kukleva, (D) a Petra Suchánková, (D) a Karel Štamberg, ${ }^{a}$ Martin Vlk, (D) a \\ Miroslav Šlouf (D) ${ }^{b}$ and Ján Kozempel (DD *a
}

We provide characterization data of hydroxyapatite ( $\mathrm{nHAp}$ ) and titanium dioxide $\left(\mathrm{nTiO}_{2}\right)$ nanoparticles as potential materials for ion sorption, e.g. in targeted therapy, barrier materials for waste repositories or photovoltaics. The study is focused on the determination of the values of protonation and ion exchange constants and site densities ( $\sum \mathrm{SOH}, \sum \mathrm{X} ;\left[\mathrm{mol} \mathrm{kg}{ }^{-1}\right]$ ) of $\mathrm{nTiO}_{2}$ and $\mathrm{nHAp}$ for further Ra kinetics and sorption experiments. These data are very important for further investigation of the materials, which can be used e.g. as drug delivery systems or in engineered barriers of deep geological repositories. The characterization was based on the evaluation of the dependence of titrating agent consumption on $\mathrm{pH}$. Titration results were evaluated on the basis of several model combinations, however the combination of the Chemical Equilibrium Model (CEM) and Ion Exchange Model (IExM) fits best to the experimental titration curves. However, the differences between the two sorbents were relatively large. Due to stability in a broad $\mathrm{pH}$ range and available surface sites, $\mathrm{nTiO}_{2}$ seems to have a wide application range. The applicability of $\mathrm{nHAp}$ is not so wide because of its dissolution under $\mathrm{pH}$ 5. Both sorbents are virtually able to sorb cationic species on deprotonated edge and layer sites with different capacities, which can be important for sorption and decontaminating applications.

Received 16th May 2019

Accepted 5th July 2019

DOI: $10.1039 / c 9 r a 03698 a$

rsc.li/rsc-advances particle and no undesirable irradiation is therefore observed. Secondly, nanoparticles can be targeted to cancer by both passive and active mechanisms, due to the EPR effect or functionalization of the surface. ${ }^{18}$ Nanohydroxyapatite and $\mathrm{nTiO}_{2}$ were chosen for this purpose because these materials are already widely used in medicine and cosmetics. They are stable, nontoxic, biocompatible, cheap, and easy to prepare. ${ }^{19-22}$ Moreover, nanoparticles can be used for theranostic systems with bonded diagnostic nuclides such as ${ }^{99 \mathrm{~m}} \mathrm{Tc},{ }^{68} \mathrm{Ga},{ }^{18} \mathrm{~F}$, etc. and therapeutic nuclides such as ${ }^{223} \mathrm{Ra},{ }^{225} \mathrm{Ac},{ }^{213} \mathrm{Bi},{ }^{186} \mathrm{Re},{ }^{90} \mathrm{Y}$, etc. ${ }^{23-28}$

Our aim was to determine nanomaterial properties including the parameters characterizing the protonation and ion-exchange processes taking place on the surface of nHAp and $\mathrm{nTiO}_{2}$ for further ${ }^{223} \mathrm{Ra}$ sorption studies, as a nanoparticle radionuclide carrier for nuclear medicine. ${ }^{29-32}$ Due to modeling of the new material surface sorption processes, separate experiments should be performed in order to obtain credible input surface protolysis and site density data.

The study is mainly focused on modelling of the sorption mechanisms. Generally, there are two types of surface groups responsible for sorption: the edge sites on the edges of the surface structure, and the layer sites which appear due to isomorphic substitution of cations and lead to a permanent negative charge on the solid particle surface. ${ }^{33,34}$ The presence of the charge depends on the crystallographic structure of a studied material, or, in some cases, on the conditions in a studied system (e.g. the surface of the material having only the
${ }^{a}$ Czech Technical University in Prague, Faculty of Nuclear Sciences and Physica Engineering, Department of Nuclear Chemistry, Břehová 7, 11519 Prague 1, Czech Republic. E-mail: jan.kozempel@fffi.cvut.cz

${ }^{b}$ Institute of Macromolecular Chemistry, Czech Academy of Sciences, Heyrovskeho nam. 2, 16206 Prague 6, Czech Republic

$\dagger$ Electronic supplementary information (ESI) available. See DOI: 10.1039/c9ra03698a 
edge sites can be contaminated with another material having the layer sites), and therefore the presence of both types of sites should be assumed.

The modeling of sorption processes requires surface protolysis and site density input data such as specific surface area, protonation and ion exchange constants, and the concentrations (densities) of edge sites and/or layer sites.

Protonation and sorption processes taking part on the edge sites can be described by several types of Surface Complexation Models (SCM) from which the following models are the most utilized: Constant Capacitance Model (CCM), Diffusion Double Layer Model (DLM), and non-electrostatistical Chemical Equilibrium Model (CEM). ${ }^{33,34}$ The processes taking part on layer sites are always described by classical Ion Exchange Model (IExM). Since hydroxyapatite can substitute $\mathrm{Ca}^{2+}$ for other cations, the necessity to involve IExM into nHAp modeling seems to be obvious. According to the literature ${ }^{35}$ the edge site density is approx. $3 \mathrm{~mol}$ $\mathrm{kg}^{-1}$ for nHAp, and approx. $0.3 \mathrm{~mol} \mathrm{~kg}^{-1}$ for $\mathrm{nTiO}_{2}$, which points to its potentially very good sorption.

General material description was performed by FTIR spectroscopy, XRPD and TEM. Detailed surface description was carried out on the basis of titration experiments through material modeling which can be briefly described as follows: the reactions taking place on the surface of nHAp or $\mathrm{nTiO}_{2}$ may be described by two equations. The first one is protonation reaction being in progress on edge sites $(\equiv \mathrm{SOH})$, the second one describes the ion-exchange on layer sites $(\equiv \mathrm{XH})$.

The balance equations of charge densities on edge sites $\left(\sum \mathrm{SOH}\right)(1)$ and layer sites $\left(\sum \mathrm{X}\right)(2)$ have to be taken into account in order to provide correct description of surface reactions:

$$
\begin{aligned}
& \Sigma \mathrm{SOH}=[\mathrm{SOH}]+\left[\mathrm{SO}^{-}\right]+\left[\mathrm{SOH}_{2}^{+}\right]\left[\mathrm{mol} \mathrm{kg}^{-1}\right] \\
& \Sigma \mathrm{X}=[\mathrm{XH}]+\left[\mathrm{X}^{-}\right]=[\mathrm{XH}]+[\mathrm{XNa}]\left[\mathrm{mol} \mathrm{kg}{ }^{-1}\right]
\end{aligned}
$$

It is important to notice that the edge sites of the nHAp are characterized as $\equiv \mathrm{POH}$ functional groups, ${ }^{36}$ while $\mathrm{nTiO}_{2}$ edge sites are formed by $\equiv \mathrm{SOH}$ groups.

Further description can be found in the ESI. $\dagger$ The description of the individual models and their application in detail were described by Filipská. ${ }^{33}$

\section{Experimental}

\section{Materials and methods}

All chemicals were of analytical grade purchased from Merck and were used without further purification: tetrabutyl ortho-titanate (TBOT), 2-propanol (IPO), sodium hydroxide, phosphoric acid, boric acid, acetic acid, nitric acid, sodium nitrate, ammonium hydroxide solution (28\%), calcium nitrate tetrahydrate, ammonium hydrogen phosphate. Demineralized water of $18 \mathrm{M} \Omega \mathrm{cm}^{-1}$ was obtained from Millipore, USA water purification system.

\section{Sorbent preparation}

nHAp. Bulk HAp was prepared by adding $1.2 \mathrm{M} \mathrm{Ca}\left(\mathrm{NO}_{3}\right)_{2}(24$ $\mathrm{mL}$ ) into $0.5 \mathrm{~L}$ of demineralized water. The $\mathrm{pH}$ of the mixture was set to 11 with ammonium hydroxide and maintained during the reaction, and $0.7 \mathrm{M}\left(\mathrm{NH}_{4}\right)_{2} \mathrm{HPO}_{4}(24 \mathrm{~mL})$ was added dropwise under stirring. The mixture was left overnight under stirring, washed with DEMI water $(3 \times 20 \mathrm{~mL})$ and dried. The resulting powder was fine-ground.

nTiO $_{2}$. The mixture of TBOT $(1 \mathrm{~mL}, 2.8 \mathrm{mmol})$ in IPO $(4 \mathrm{~mL})$ was dropwise added to $100 \mathrm{~mL}$ of DEMI water in ultrasonic generator. The suspension was stirred for 30 minutes at the laboratory temperature. Prepared nanoparticles were washed with DEMI water $(3 \times 20 \mathrm{~mL})$, once with IPO $(10 \mathrm{~mL})$ and then dried under vacuum. The resulting powder was fine-ground.

\section{Characterization of the materials}

The specific surface area was determined by selective sorption of nitrogen at the temperature of liquid nitrogen using a multipoint BET method. Confirmation of the composition of the materials was made with FTIR spectra recorded on Nicolet iS50 FTIR (ThermoScientific, USA) in middle infrared region 400$4000 \mathrm{~cm}^{-1}$ with resolution $2 \mathrm{~cm}^{-1}$ on a diamond crystal which were compared to relevant records in HR Inorganics I. Minerals database. ${ }^{37}$ Powder X-ray diffractograms using Rigaku MiniFlex 600 (Ni-filtered $\mathrm{Cu}-\mathrm{K}_{\alpha 1,2}$ radiation) equipped with $\mathrm{NaI}(\mathrm{Tl})$ scintillation detector were compared to the relevant records in the ICDD PDF-2 database (version 2013). ${ }^{38}$

TEM analyses. The nanoparticles were dispersed in water, a small droplet $(2 \mu \mathrm{L})$ of the suspension was dropped onto the standard TEM supporting copper grid covered with an electron transparent carbon film. The excess of the solution was removed after $1 \mathrm{~min}$ by touching the bottom of the grid by a thin strip of filter paper in order to avoid oversaturation and nanoprecipitation during the drying process. The specimens were then left to dry completely. After drying the samples were transferred to a TEM microscope (Tecnai G2 Spirit Twin 12; FEI Company, Czech Republic) and observed at accelerating voltage $120 \mathrm{kV}$. Morphology of the particles was observed with standard bright field imaging (TEM/BF), energy-dispersive analysis of Xrays (TEM/EDX) yielded the elemental composition, and selected area electron diffraction (TEM/SAED) was used to verify the crystalline structures. Electron diffraction patterns were transformed to 1D-diffractograms (by means of Process Diffraction $^{39}$ ) and compared with X-ray diffraction patterns (calculated with PowderCell ${ }^{40}$ ) of the expected crystalline structures of nHAp and $\mathrm{nTiO}_{2}$ (the structures were obtained from Crystallography Open Database ${ }^{\mathbf{4 1}}$ ). An estimate of crystallite size from electron diffraction patterns and a calculation of particle size distribution and specific surface area in comparison with BET experiment is provided in ESI. $\dagger$

\section{Potentiometric titrations}

Automatic titration was made on TIM845 Potentiometric Titrator (HACH, USA) equipped with $\mathrm{Ag} / \mathrm{AgCl}$ electrode (HACH, USA).

The nHAp or $\mathrm{nTiO}_{2}(150 \mathrm{mg})$ was dispersed in $50 \mathrm{~mL}$ of $0.1 \mathrm{M}$ $\mathrm{NaNO}_{3}$ to ensure stability of the ionic strength (suspension concentration was $3 \mathrm{~g} \mathrm{~L}^{-1}$ ). Titration was performed at $23 \pm 1{ }^{\circ} \mathrm{C}$ with $0.1 \mathrm{M} \mathrm{NaOH}$ for alkaline part of titration curve and $0.1 \mathrm{M}$ $\mathrm{HNO}_{3}$ for acidic one. During the titration with $\mathrm{NaOH}$ the sample 

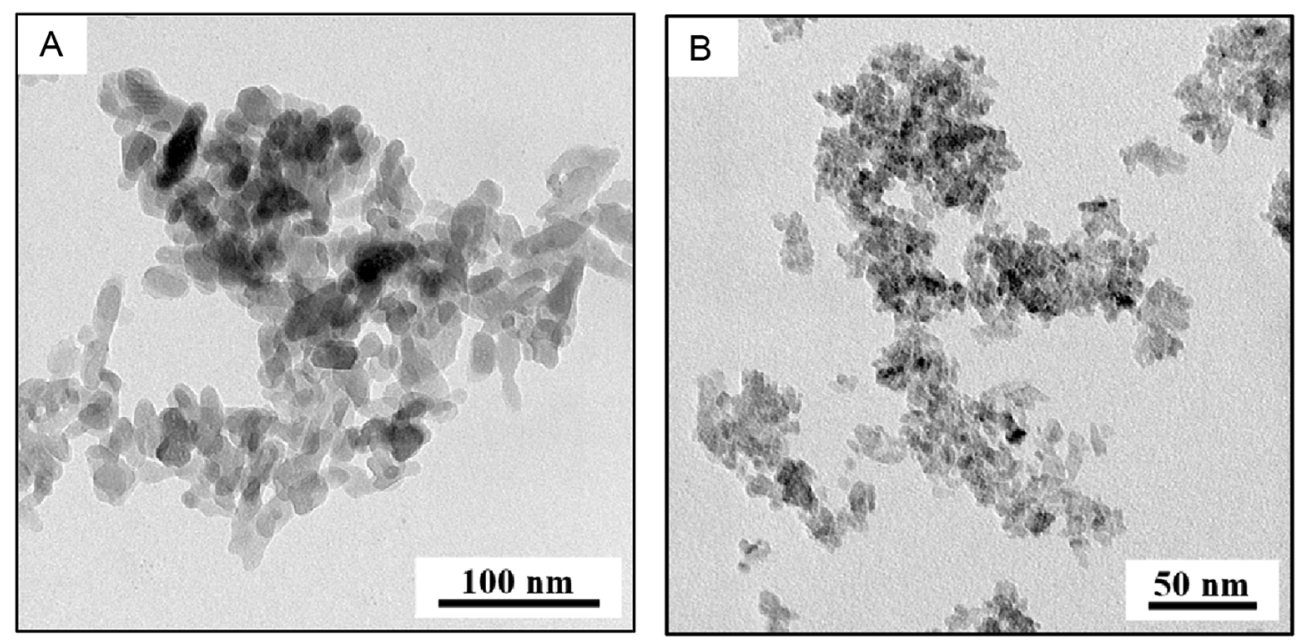

Fig. 1 TEM analysis (A) TEM/BF micrograph showing the size and shape of $n H A p$, (B) TEM/BF micrograph showing the size and shape of $n$ TiO 2 .

and the titrant were bubbled with $\mathrm{N}_{2}$ to eliminate atmospheric $\mathrm{CO}_{2}$ absorption and dissolution.

In the case of $\mathrm{nTiO}_{2}$, the studied $\mathrm{pH}$ range was between 2.5 and 10. In the case of nHAp, with regard to its possible dissolving in acidic medium, the lowest reached $\mathrm{pH}$ was approx. 5. The experiments were repeated three times for each line and solid phase. A blank experiment was performed with $50 \mathrm{~mL}$ of $0.1 \mathrm{M} \mathrm{NaNO}_{3}$ without any solid phase added for the both acidic and alkaline part of the titration curve. Increment was set to $0.05 \mathrm{~mL}$ and stability conditions to $20 \mathrm{mpH} \mathrm{min}^{-1}$. The $\mathrm{pH}$ was recorded after each addition of titrant as a function of its volume.

\section{Results and discussion}

\section{Specific surface area, FTIR, XRPD}

The specific surface areas via BET measurements were determined at $117 \pm 8 \mathrm{~m}^{2} \mathrm{~g}^{-1}$ for nHAp and $330 \pm 10 \mathrm{~m}^{2} \mathrm{~g}^{-1}$ for $\mathrm{nTiO}_{2}$. FTIR spectra of the nHAp showed the characteristic vibration bands of hydroxyapatite: vibration bands at $600-473 \mathrm{~cm}^{-1}$ and 1089$962 \mathrm{~cm}^{-1}$ might be attributed to $\mathrm{PO}_{4}{ }^{3-}$. Band at $\sim 1400 \mathrm{~cm}^{-1}$ belongs to the characteristic vibration of the $\mathrm{CO}_{3}{ }^{2-}$, that at $1630 \mathrm{~cm}^{-1}$ corresponds to deformation vibration band of $\mathrm{OH}^{-}$and that at $3500-3000 \mathrm{~cm}^{-1}$ belongs to stretching mode of $\mathrm{OH}^{-}$. FTIR spectrum of $\mathrm{nTiO}_{2}$ has the band at $3500-3000 \mathrm{~cm}^{-1}$ that might be attributed to stretching mode of $\mathrm{OH}^{-}$and at $1632 \mathrm{~cm}^{-1}$ associated with deformation vibrations of $\mathrm{OH}^{-}$bonds on the surface of $\mathrm{TiO}_{2}$. Arms on the band at $1000-400 \mathrm{~cm}^{-1}$ are assigned to Ti-O and TiO-Ti stretching modes. ${ }^{42}$ Both FTIR spectra were compared to database ${ }^{37}$ records \#15 and \#216 for nHAp and $\mathrm{nTiO}_{2}$ respectively; they fit properly.

Nanohydroxyapatite and $\mathrm{nTiO}_{2}$ diffractograms showed that both samples are single phase. Experimental conditions for nHAp synthesis meet the requirements for hydroxyapatite formation. Titanium dioxide has the structure of anatase as proved by library data ${ }^{38}$ comparison. Low intensity peaks of $\mathrm{nTiO}_{2}$ can be interpreted either as nano-sized crystals or as amorphous phase formation. According to TEM/BF micrograph (Fig. 1B) the nanoparticle size is under $20 \mathrm{~nm}$.

Both FTIR and XRPD spectra could be found in the ESI (Fig. $\mathrm{S} 1$ and $\mathrm{S} 2 \dagger$ ).
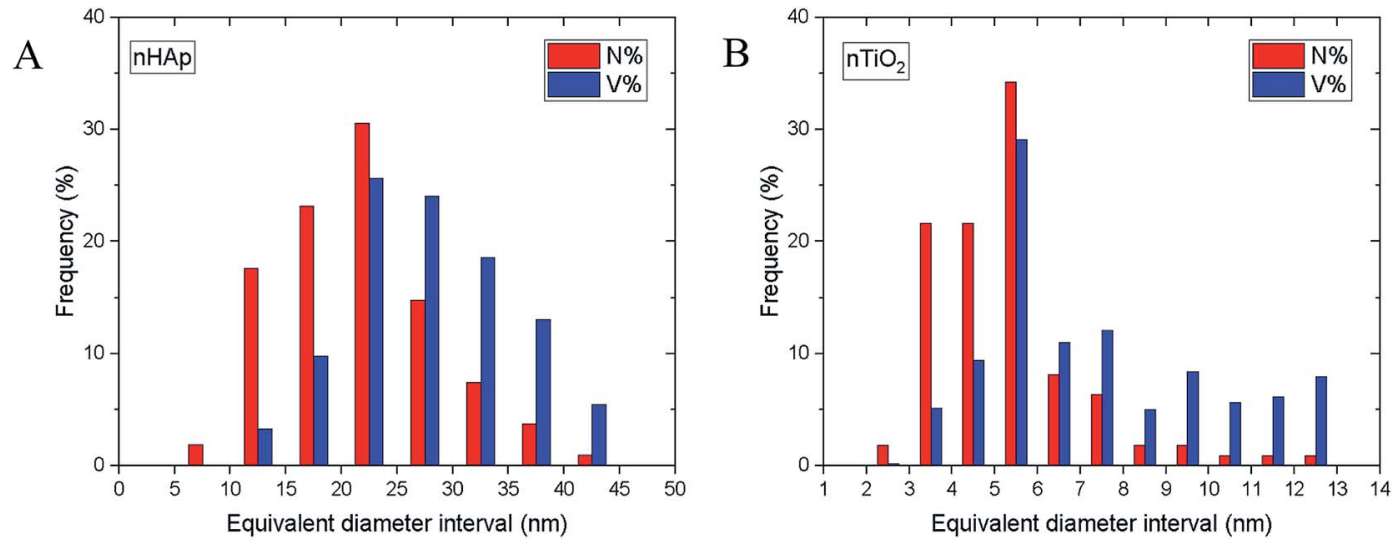

Fig. 2 Particle size distributions determined from image analysis of TEM/BF micrographs using program MDISTR ${ }^{45-47}$ for (A) $n H_{A p}$ and (B) $n$ TiO 2 . The symbols N\% and V\% denote number and volume distributions, respectively. 
Table 1 Morphological descriptors of the nanoparticles

\begin{tabular}{llllllll}
\hline & & \multicolumn{2}{c}{$\begin{array}{l}\text { Equivalent } \\
\text { diameter }[\mathrm{nm}]\end{array}$} & & \multicolumn{2}{c}{ Elongation $[-]$} \\
\cline { 3 - 4 } Sample & Crystallite size $^{a}[\mathrm{~nm}]$ & Average & StDev & & Average & StDev \\
\hline nHAp & 5.18 & 21.7 & 6.9 & & 1.70 & 0.49 \\
nTiO $_{2}$ & 2.44 & 5.3 & 1.7 & & 1.50 & 0.41
\end{tabular}

${ }^{a}$ Crystallite size was obtained from TEM/SAED diffractograms using Scherrer equation. Equivalent diameter and elongation were assessed from measurements of TEM/BF micrographs.

\section{TEM characterization of the nanoparticles}

TEM characterization of nHAp and $\mathrm{nTiO}_{2}$ is shown in Fig. 1 where TEM/BF micrographs demonstrated that both nanoparticles had quite narrow size distribution, nHAp (Fig. 1A) being larger than $\mathrm{nTiO}_{2}$ (Fig. 1B). TEM/SAED diffractograms and their comparison with theoretically calculated XRD diffraction patterns confirmed the expected crystalline structures: hexagonal structure of nHAp (Crystallography Open Database $^{41}$ COD \#9001233) and tetragonal anatase structure of $\mathrm{nTiO}_{2}$ (Crystallography Open Database ${ }^{41}$ COD \#1526931). TEM/ EDX spectra yielded the expected elemental composition and confirmed that both prepared nanoparticles were free from impurities (we note that $\mathrm{C}$ and $\mathrm{Cu}$ peaks come from the standard TEM supporting copper grids, which were covered with thin, electron transparent carbon film). TEM/SAED diffractograms and TEM/EDX spectra are shown in ESI (Fig. S3 and S4†).

\section{TEM image analysis and calculation of specific surface area}

Both TEM/BF micrographs and TEM/SAED diffraction patterns were employed in estimating the nanoparticle size. Image analysis

Table 2 Particle numbers and specific surface areas calculated by the MDISTR program

\begin{tabular}{llllll}
\hline & nHAp & & & $\mathrm{nTiO}_{2}$ \\
\cline { 2 - 3 } \cline { 5 - 6 } Model ID $^{a}$ & $N[-]$ & $A\left[\mathrm{~m}^{2} \mathrm{~g}^{-1}\right]$ & & $N[-]$ & $A\left[\mathrm{~m}^{2} \mathrm{~g}^{-1}\right]$ \\
\hline Model 1 & $4.35 \times 10^{18}$ & 367 & $3.11 \times 10^{19}$ & 581 \\
Model 2 & $5.92 \times 10^{16}$ & 88 & $3.05 \times 10^{18}$ & 268 \\
Model 3 & $4.49 \times 10^{16}$ & 73 & $2.25 \times 10^{18}$ & 218 \\
Model 4 & $8.98 \times 10^{16}$ & 102 & & $4.49 \times 10^{18}$ & 298 \\
Model 5 & $8.98 \times 10^{16}$ & 107 & $4.49 \times 10^{18}$ & 342 \\
BET experiment & - & 117 & - & 330
\end{tabular}

${ }^{a}$ All calculations were performed by the MDISTR $^{45-47}$ program based on parameters from image analysis of TEM/BF micrographs and/or TEM/ SAED diffraction patterns. $N$ denotes number of particles and $A$ denotes specific surface area of the nanoparticles. The models used for calculations were as follows: Model $1=$ uniform spherical particles with average size determined from broadening of TEM/SAED diffractions (5.2 nm for nHAp, $2.4 \mathrm{~nm}$ for $\mathrm{nTiO}_{2}$ ); Model $2=$ uniform spherical particles with average size determined from image analysis of TEM/BF micrographs (21.7 nm for nHAp, $5.3 \mathrm{~nm}$ for $\mathrm{nTiO}_{2}$ ); Model $3=$ spherical particles with the size distribution determined from image analysis; Model $4=$ the same as previous, but with additional corrections for elongation and flatness of the particles, which were measured or estimated from TEM micrographs; Model $5=$ the same as previous, but with additional correction for surface roughness of the nanoparticles, which was estimated from TEM micrographs.

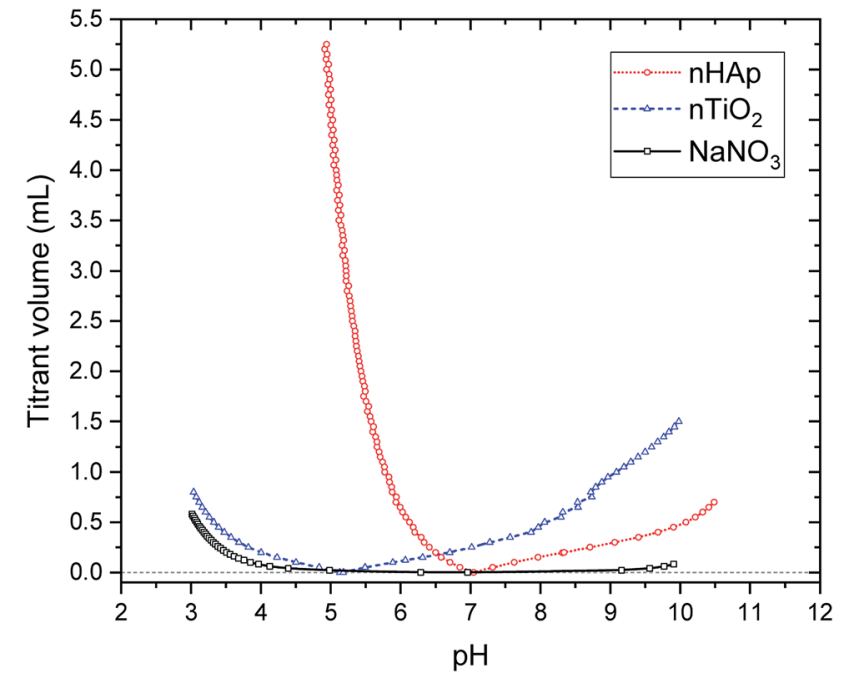

Fig. 3 Experimental dependences of added volume of titrant $0.1 \mathrm{M}$ $\mathrm{NaOH}$, for basic part and $0.1 \mathrm{M} \mathrm{HNO}_{3}$ for acidic part of titration curves on $\mathrm{pH}$ and blank titration curve of $0.1 \mathrm{M} \mathrm{NaNO}_{3}$.

of TEM/BF micrographs was based on direct measurement of $>100$ particles and yielded an average particle size (morphological descriptor: equivalent diameter), aspect ratios (morphological descriptor: elongation) and the particle size distributions (shown in Fig. 2); technical details concerning image analysis are given in the ESI. $\dagger$ An estimate of the nanoparticle sizes from TEM/SAED was based on Scherrer equation. ${ }^{43}$ It is worth noting that Scherrer equation is applied mostly to X-ray diffraction data, but its application on SAED diffraction patterns is also possible, although it may not be so common. ${ }^{\mathbf{4 3 , 4 4}}$ The average particle sizes are summarized in Table 1.

The nHAp and $\mathrm{nTiO}_{2}$ morphological descriptors (Table 1), particle size distributions (Fig. 2) and overall semi-quantitative characteristics of the observed nanoparticles (such as their roughness estimated from their shapes in higher magnification TEM/BF micrographs), were employed in the calculation of the specific surface area of the nanoparticles. The calculations, which are properly described in the ESI, $\uparrow$ were performed by our program MDISTR. ${ }^{45-47}$ The final calculated values were compared with the experimentally determined specific surface

Table 3 Evaluation of titration curves by six different models: the values of WSOS/DF characterizing the agreement between the experimental $\left(22 \pm 1^{\circ} \mathrm{C}\right)$ and calculated data (N/A - evaluation was not performed)

\begin{tabular}{lll}
\hline & \multicolumn{1}{c}{ nHAp } & $\mathrm{nTiO}_{2}$ \\
\cline { 2 - 2 } Model & WSOS/DF & WSOS/DF \\
\hline CEM & 34.1 & 8.88 \\
CEM + IExM & 1.07 & 1.26 \\
CCM & 46.9 & 1.77 \\
CCM + IExM & 42.4 & 1.88 \\
DLM & N/A & 1.16 \\
DLM + IExM & 13.4 & 0.70
\end{tabular}


Table 4 The total $n \mathrm{HAp}$ concentration of edge sites ( $\left.\sum \mathrm{POH}\right)$ and ion exchange groups (layer sites) $\left(\sum \mathrm{X}\right)$, protonation $\left(K_{1}, \mathrm{~K}_{2}\right)$, and ion exchange $\left(\mathrm{K}_{\mathrm{Na}^{+} / \mathrm{H}^{+}}\right)$constants based on the titration curve evaluation

\begin{tabular}{|c|c|c|c|c|c|}
\hline Model & $\sum \mathrm{POH}\left[\mathrm{mol} \mathrm{kg}^{-1}\right]$ & $\sum \mathrm{x}\left[\mathrm{mol} \mathrm{kg}{ }^{-1}\right]$ & $K_{1}\left[\mathrm{~L} \mathrm{~mol}^{-1}\right]$ & $K_{2}\left[\mathrm{~L} \mathrm{~mol}^{-1}\right]$ & $K_{\mathrm{Na}^{+} / \mathrm{H}^{+}}[-]$ \\
\hline CEM + IEXM & $5.10 \pm 1.20$ & $0.15 \pm 0.01$ & $5.12 \times 10^{11} \pm 1.10 \times 10^{11}$ & $1.19 \times 10^{5} \pm 2.73 \times 10^{4}$ & $3.01 \times 10^{6} \pm 3.26 \times 10^{5}$ \\
\hline DLM + IExM & $13.90 \pm 4.35$ & $0.20 \pm 0.10$ & $1.83 \times 10^{11} \pm 6.84 \times 10^{10}$ & $1.80 \times 10^{7} \pm 5.07 \times 10^{6}$ & $1.12 \times 10^{5} \pm 3.34 \times 10^{5}$ \\
\hline
\end{tabular}

Table 5 The total $n \mathrm{niO}_{2}$ concentration of edge sites $\left(\sum \mathrm{SOH}\right)$ and ion exchange groups (layer sites) $\left(\sum \mathrm{X}\right)$, protonation $\left(K_{1}, K_{2}\right)$, and ion exchange $\left(\mathrm{KNa}^{+} / \mathrm{H}^{+}\right)$constants based on evaluation of titration curves

\begin{tabular}{llllll}
\hline Model & $\sum \mathrm{SOH}\left[\mathrm{mol} \mathrm{kg}^{-1}\right]$ & $\sum \mathrm{X}\left[\mathrm{mol} \mathrm{kg}{ }^{-1}\right]$ & $K_{1}\left[\mathrm{~L} \mathrm{~mol}^{-1}\right]$ & $K_{2}\left[\mathrm{~L} \mathrm{~mol}^{-1}\right]$ & $K_{\mathrm{Na}^{+} / \mathrm{H}^{+}}[-]$ \\
\hline CEM + IExM & $0.20 \pm 0.01$ & $0.67 \pm 0.01$ & $2.31 \times 10^{6} \pm 1.93 \times 10^{4}$ & $1.84 \times 10^{4} \pm 1.65 \times 10^{2}$ & $5.67 \times 10^{7} \pm 2.01 \times 10^{6}$ \\
DLM + IExM & $1.42 \pm 0.10$ & $0.07 \pm 0.01$ & $3.26 \times 10^{7} \pm 2.73 \times 10^{6}$ & $4.32 \times 10^{3} \pm 2.61 \times 10^{2}$ & $6.96 \times 10^{4} \pm 1.74 \times 10^{3}$ \\
\hline
\end{tabular}
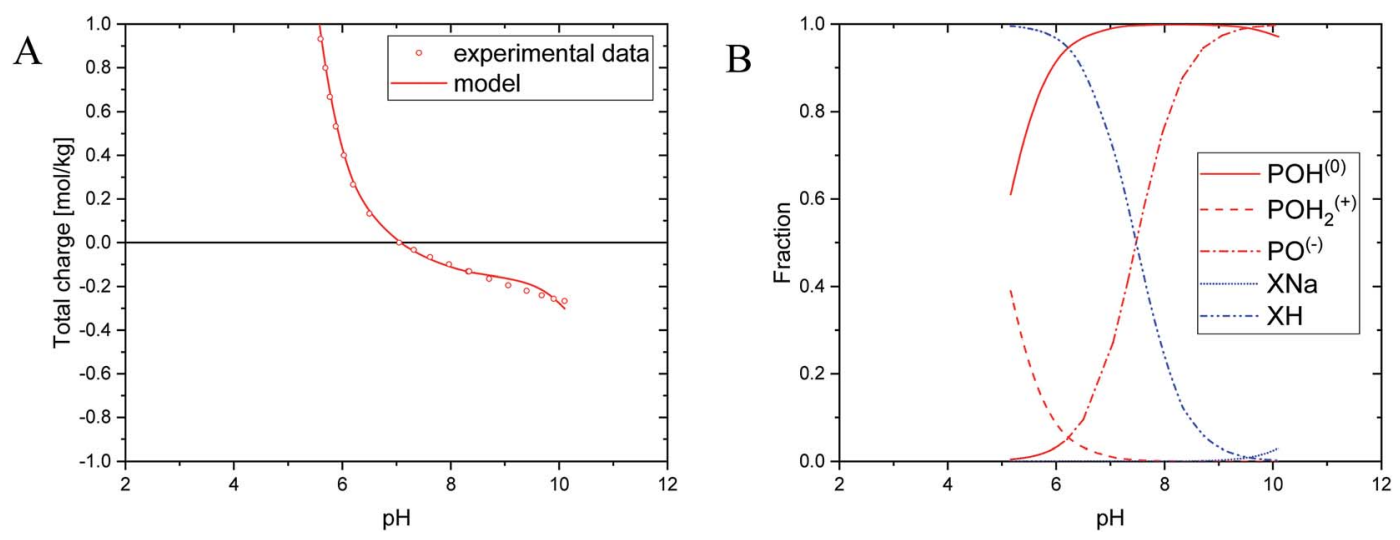

Fig. 4 (A) Experimental data and calculated titration curve; (B) molar fractions of individual forms of surface sites for nHAp (CEM + IExM).

areas from BET experiments (Table 2). The agreement between theoretical calculations based on TEM data including all corrections and the experimental results from BET experiments was very good. In both samples, the SAED-based crystallite sizes resulted in overestimation of specific surface area in comparison with BET experiments (Table 2, compare results of Model 1 and BET experiments), which implied that SAED-based crystallite sizes were small, resulting in too high numbers of particles with too high specific surface area. Other models showed good agreement with BET experimental results. For more information see ESI. $\dagger$
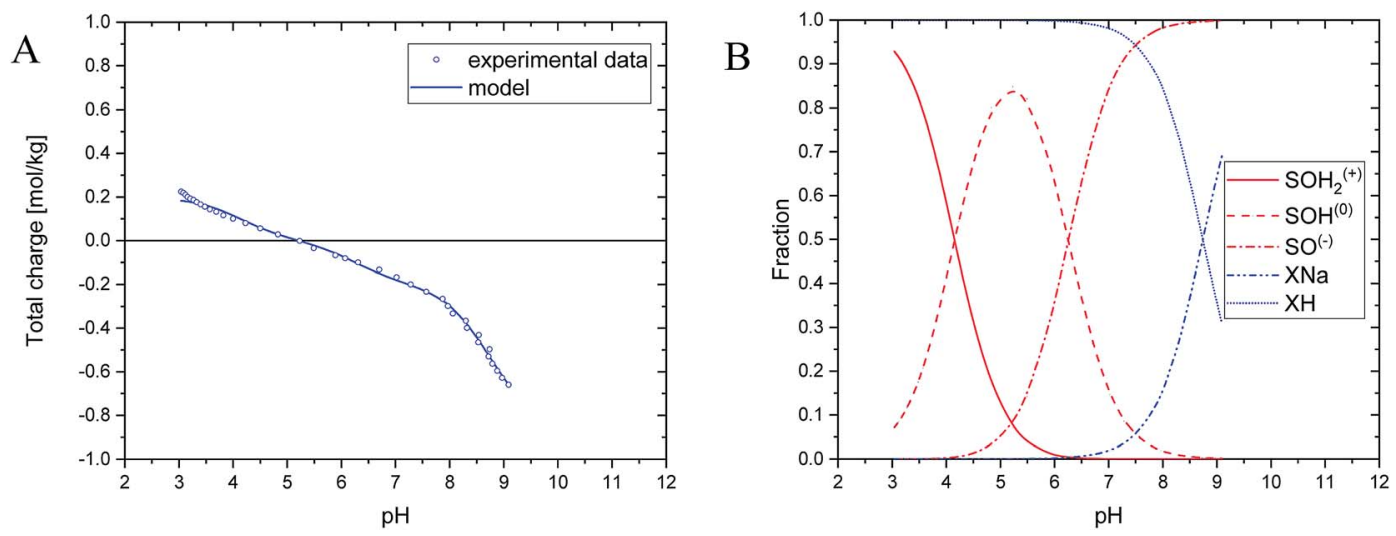

Fig. 5 (A) Experimental and calculated titration curve; (B) molar fractions of individual forms of surface sites for $n T i O_{2}(C E M+I E x M)$. 


\section{Evaluation of the titration experiments}

Experimentally obtained dependences are shown in Fig. 3. As mentioned above, due to nHAp dissolution the titration range was from 5 to 10 . Strictly speaking, the dissolution of nHAp starts at aprox. $\mathrm{pH}=7$, but it is negligible if the titration is fast enough and $\mathrm{pH}=5$ is reached within a few minutes.

The WSOS/DF value (weighted sum of squares of differences divided by the degrees of freedom) is taken as an appropriate criterion of goodness-of-fit and it should be in the interval $0.1<$ WSOS/DS $<20$, so the model used for the description of the given experimental dependence can be taken as acceptable. In addition, the physical meaning of individual parameter values has to be taken into account, as well. Evidently, the WSOS/DF criterion (Table 3) is completely fulfilled for $\mathrm{nTiO}_{2}$, for nHAp it holds for CEM + IExM and DLM + IExM models, only. But, if we take into account the values in Tables 4 and 5, and if we allow for literature data $\sum \mathrm{SOH}$ and $\sum \mathrm{POH}$ mentioned above, ${ }^{35}$ combination of CEM + IExM models describe experimental data in the most accurate way for both nHAp and $\mathrm{nTiO}_{2}$.

According to the chosen models, total concentrations of the edge sites and ion exchange groups, protonation and ion exchange constants (for $\mathrm{Na}^{+} / \mathrm{H}^{+}$exchange) were calculated and their values were summarized in Table 4 for nHAp, and in Table 5 for $\mathrm{nTiO}_{2}$. On comparing the parameter values in Tables 1-3 to each other and considering the literature data mentioned above, ${ }^{35}$ the best model seems to be CEM + IExM for both materials.

The experimental and calculated titration curves are depicted as total surface charge density, $Q\left[\mathrm{~mol} \mathrm{~kg}^{-1}\right]$,or $\left(Q_{\text {cal }}\right)_{\mathrm{i}}$, on $\mathrm{pH}$ for nHAp and $\mathrm{nTiO}_{2}$, respectively (Fig. $4 \mathrm{~A}$ and $5 \mathrm{~A}$ ). The molar fractions of individual forms of sites (see the ESI $\dagger$ ) can be found, again as the dependences on $\mathrm{pH}$ (Fig. 4B and 5B). In both cases, CEM + IExM model was used for experimental data evaluation.

Of course, there are great differences between nHAp and $\mathrm{nTiO}_{2}$ not only in the density values of surface sites $\left(\sum \mathrm{SOH}\right.$, $\sum \mathrm{POH}$ and $\left.\sum \mathrm{X}\right)$, but above all in the values of protonation constants - what does it means, it is evident from the comparison of curves in Fig. 4B and 5B. Evidently, the pH value plays the basic role in the applicability of both materials to the surface sorption of anionic, cationic and molecular species (surface complexation). The applicability of nHAp is limited to pH interval from approx. 5 to 11 , whereas in case of $\mathrm{nTiO}_{2}$, it deals with $\mathrm{pH}$ from approx. 3 to 11 . The both materials differ in the sorption property (ability) in respect of different species, such that on nHAp (Fig. 4B) can take place the sorption of cationic forms on $\mathrm{PO}^{-}$and $\mathrm{X}^{-}$or surface complexation can proceeds on $\mathrm{PO}^{-}$. As for $\mathrm{nTiO}_{2}$ (Fig. 5B), cationic forms can be sorbed on $\mathrm{SO}^{-}$and $\mathrm{X}^{-}$, and by analogy the surface complexation can proceed on $\mathrm{SO}^{-}$, in addition, the sorption of anionic species can be under way on $\mathrm{SOH}_{2}{ }^{+}$. Evidently, in principle, both materials can be used as carrier of cation in question, i.e., ${ }^{223} \mathrm{Ra}$, namely, for example as ${ }^{223} \mathrm{Ra}^{2+}$ or ${ }^{223} \mathrm{Ra}(\mathrm{OH})^{+}$.

\section{Conclusions}

An investigation of nHAp and $\mathrm{nTiO}_{2}$ preparation and surface sorption properties was performed in order to provide more detailed data for further studies considering ${ }^{223} \mathrm{Ra}$ uptake on these sorbents. However, the differences between the two sorbents were relatively large, as follows from the values of parameters determined for nHAp $\left(K_{1}=5.12 \times 10^{1}\left[\mathrm{~L} \mathrm{~mol}^{-1}\right] ; K_{2}\right.$ $=1.19 \times 10^{5}\left[\mathrm{~L} \mathrm{~mol}^{-1}\right] ; \sum \mathrm{SOH}=5.10\left[\mathrm{~mol} \mathrm{~kg}^{-1}\right] ; K_{\mathrm{ex}}=3.01 \times$ $\left.10^{6}[-] ; \sum \mathrm{X}=0.15\left[\mathrm{~mol} \mathrm{~kg}^{-1}\right]\right)$, and for $\mathrm{nTiO}_{2}\left(K_{1}=2.31 \times 10^{6}\right.$ $\left[\mathrm{L} \mathrm{mol}^{-1}\right] ; K_{2}=1.84 \times 10^{4}\left[\mathrm{~L} \mathrm{~mol}^{-1}\right] ; \sum \mathrm{SOH}=0.20\left[\mathrm{~mol} \mathrm{~kg}^{-1}\right] ;$ $\left.K_{\text {ex }}=5.67 \times 10^{7}[-] ; \sum \mathrm{X}=0.67\left[\mathrm{~mol} \mathrm{~kg}{ }^{-1}\right]\right)$. Deep understanding of the surface properties of $\mathrm{HAp}$ and $\mathrm{TiO}_{2}$ nanoparticles, as prospective carriers for targeted alpha-particle therapy by in vivo generators, is important. ${ }^{18}$ The results will allow us to predict and provide further evaluation of kinetics and sorption dependences of various ions and radionuclides, including ${ }^{223} \mathrm{Ra}$ and its decay products which are present in the form of cations. Therefore, the studied nanoparticles, which are capable of both capture and resorption, due to their high capacity and suitable size, are a promising solution.

Based on our experiments and calculations it can be stated that applicability of $\mathrm{nTiO}_{2}$ is high due to its stability in a broad $\mathrm{pH}$ range and available surface sites $\left(\sum \mathrm{SOH}=0.20 \mathrm{~mol} \mathrm{~kg}^{-1}\right.$; $\sum \mathrm{X}=0.67 \mathrm{~mol} \mathrm{~kg}^{-1}$ ). Evidently, cationic species sorption on $\mathrm{nTiO}_{2}$ can proceed via ion-exchange on deprotonated edge sites $\left(\equiv \mathrm{SO}^{-}\right)$and on layer sites $\left(\equiv \mathrm{X}^{-}\right)$as well. Applicability of nHAp is not as wide as $\mathrm{nTiO}_{2}$ because of its dissolution under pH 5 . On the one hand, surface site density of nHAp is greater than that of $\mathrm{nTiO}_{2}\left(\sum \mathrm{POH}=5.10 \mathrm{~mol} \mathrm{~kg}^{-1} ; \sum \mathrm{X}=0.15 \mathrm{~mol} \mathrm{~kg}^{-1}\right)$, which can be regarded as an advantage of nHAp. On the other hand, nHAp deprotonated edge sites $\left(\equiv \mathrm{PO}^{-}\right)$are virtually able to sorb only cationic species. Uptake of anionic and molecular species on edge sites of the two sorbents can also undergo surface complexation mechanism, but regarding the purposes of our study it was not taken into account.

\section{Conflicts of interest}

There are no conflicts to declare.

\section{Acknowledgements}

Research at the Czech Technical University was supported by the grants of NV16-30544A (the Health Research Agency of the Czech Republic), CZ.02.1.01/0.0/0.0/15_003/0000464 (EU and the Ministry of Education, Youth and Sports of the Czech Republic), and SGS16/251/OHK4/3T/14 (Czech Technical University in Prague). Electron microscopy at the Institute of Macromolecular Chemistry was supported through grants 1705007S (Czech Science Foundation) and POLYMAT LO1507 (Ministry of Education, Youth and Sports of the CR, program NPU I).

\section{References}

1 T. Jones and T. A. Egerton, Titanium Compounds, Inorganic, in Kirk-Othmer Encyclopedia of Chemical Technology, John Wiley \& Sons, New York, 2000.

2 R. Z. LeGeros, A. Ito, A. Ishikawa, T. Sakae and J. P. LeGeros, in Advanced Biomaterials: Fundamentals, Processing, and 
Applications, The American Ceramic Society, Ohio, 2010, ch. 2, pp. 19-52.

3 S. A. Pappus, B. Ekka, S. Sahu, D. Sabat, D. Dash and M. Mishra, J. Nanopart. Res., 2017, 19(4), 136.

4 C. Bogatu, D. Perniu, C. Sau, O. Iorga, M. Cosnita and A. Duta, Ceram. Int., 2017, 43(11), 7963-7969.

5 A. Corami, S. Mignardi and V. Ferrini, J. Hazard. Mater., 2007, 146(1-2), 164-170.

6 S. Handley-Sidhu, T. K. Mullan, Q. Grail, M. Albadarneh, T. Ohnuki and L. E. Macaskie, Sci. Rep., 2016, 6(1), 23361.

7 L. Reddy, P. Venkata, B. Kavitha, P. Anil, K. Reddy and K. H. Kim, Environ. Res., 2017, 154, 296-303.

8 H. He, B. Wu and C. Yang, Int. J. Environ. Sci. Technol., 2018, 15, DOI: $10.1007 / \mathrm{s} 13762-018-2148-2$.

9 E. Cędrowska, M. Pruszynski, A. Majkowska-Pilip, S. Męczyńska-Wielgosz, F. Bruchertseifer, A. Morgenstern and A. Bilewicz, J. Nanopart. Res., 2018, 20(3), 83.

10 D. Duan, H. Liu, Y. Xu, Y. Han, M. Xu, Z. Zhang and Z. Liu, ACS Appl. Mater. Interfaces, 2018, 10(6), 5278-5286.

11 A. Rajeswari, K. V. Vimalnath, H. D. Sarma, P. Shetty, S. K. Mohammed, J. Nuwad, S. Chakraborty and A. Dash, Appl. Radiat. Isot., 2017, 116, 85-91.

12 K. V. Vimalnath, S. Chakraborty, A. Rajeswari, H. D. Sarma, J. Nuwad, U. Pandey, K. Kamaleshwaran, A. Shinto and A. Dash, Nucl. Med. Biol., 2015, 42(5), 455-464.

13 X. Wu, W. Qin, S. Wang, Z. Jiang, Y. Guo and Z. Xie, Rare Met., 2006, 25(6), 169-172.

14 P. Mazierski, A. Mikolajczyk, B. Bajorowicz, A. Malankowska, A. Zaleska-Medynska and J. Nadolna, Appl. Catal., B, 2018, 233, 301-317.

15 S. Mondal, S. V. Dorozhkin and U. Pal, Wiley Interdiscip. Rev.: Nanomed. Nanobiotechnol., 2018, 10, e1504.

16 O. Kaygili, S. Keser, S. V. Dorozhkin, F. Yakuphanoglu, A. A. Al-Ghamdi, S. Kirbag, D. Sertkaya, T. Ates and N. Canan Gursoy, J. Inorg. Organomet. Polym., 2014, 24, 1001-1008.

17 J. Kozempel, M. Vlk, E. Malková, A. Bajzíková, J. Bárta, R. Santos-Oliveira and A. Malta Rossi, J. Radioanal. Nucl. Chem., 2015, 304, 443-447.

18 J. Kozempel, O. Mokhodoeva and M. Vlk, Molecules, 2018, 23(3), 581.

19 M. P. Ferraz, F. J. Monteiro and C. M. Manuel, J. Appl. Biomater. Funct. Mater., 2004, 2(2), 74-80.

20 S. Koutsopoulos, J. Biomed. Mater. Res., 2002, 62(4), 600-612.

21 M. Malekshahi Byranvand, A. Nemati Kharat, L. Fatholahi and Z. Malekshahi Beiranvand, J. Nanostruct., 2013, 3, 1-9.

22 S. Mital Gupta and M. Tripathi, Cent. Eur. J. Chem., 2012, 10(2), 279-294.

23 L. D. Esposti, A. Tampieri and M. Iafisco, in Nanotechnologies in Preventive and Regenerative Medicine, ed. V. Uskokovic, Elsevier, New York, 2017, ch. 6.3, pp. 465-486.

24 W. Zhou and J. Zheng, Adv. Mat. Res., 2012, 503/504, 688691.
25 S. Chakraborty, K. V. Vimalnath, A. Rajeswari, H. D. Sarma, A. Shinto, E. R. Radhakrishnan and A. Dash, J. Radioanal. Nucl. Chem., 2017, 302(2), 875-881.

26 J. Xie, S. Lee and X. Chen, Adv. Drug Delivery Rev., 2010, 62(11), 1064-1079.

27 M. Sakmar, M. Vlk, P. Suchankova, E. Kukleva, J. Kozempel, M. Hruby and V. Lobaz, presented in part at $13^{\text {th }}$ international Symposium on the Synthesis and Application of Isotopically Labelled Compounds, Prague, June, 2018.

28 P. Micolova, E. Kukleva, P. Nykl, M. Sakmar, M. Vlk, L. Nespesna and J. Kozempel, presented in part at $22^{\text {nd }}$ International Symposium on Radiopharmaceutical Sciences, Dresden, May, 2017.

29 Xofigo - summary of product characteristics, http:// www.ema.europa.eu/docs/en_GB/document_library/EPAR__Product_Information/human/002653/WC500156172.pdf, accessed January 2018.

30 S. Mirzadeh, Appl. Radiat. Isot., 1998, 49(4), 345-349.

31 E. Kukleva, J. Kozempel, M. Vlk, P. Micolova and D. Vopalka, J. Radioanal. Nucl. Chem., 2014, 304(1), 263-266.

32 O. Mokhodoeva, L. Guseva and N. Dogadkin, J. Radioanal. Nucl. Chem., 2014, 304(1), 449-453.

33 H. Filipská and K. Štamberg, Acta Polytech., 2005, 45(5), 1118.

34 J. Lützenkirchen, Surface complexation modelling, Academic Press, Elsevier Ltd., London, 2006.

$35 \mathrm{RES}^{3} \mathrm{~T} /$ Rossendorf Expert System for Surface and Sorption Thermodynamics, https:/www.hzdr.de/db/res3t.login, accessed January 2018.

36 B. Sandrine, N. Ange, B. A. Didier, C. Eric and S. Patrick, J. Hazard. Mater., 2007, 139(3), 443-446.

37 HR Inorganics I. - Minerals database - FTIR, Thermo Scientific $^{\mathrm{TM}}$ OMNIC ${ }^{\mathrm{TM}}$, USA.

38 ICDD PDF-2 database, Version 2013, ISDD, USA.

39 J. L. Lábár, Ultramicroscopy, 2005, 103, 237-249.

40 W. Kraus and G. Nolze, J. Appl. Crystallogr., 1996, 29, 301303.

41 Crystallography Open Database-an open-access collection of crystal structures, http://www.crystallography.net/cod/ search.html, accessed May 2018.

42 T. Ivanova and A. Harizanova, Solid State Ionics, 2001, 138, 227-232.

43 B. Ingham and M. F. Toney, Chapter 1: X-ray diffraction for characterizing metallic films, in Metallic Films for Electronic, Optical and Magnetic Application, ed. K. Barmak and K. Coffey, Elsevier, New York, 2014, pp. 3-38.

44 B. Fultz and J. Howe, Transmission electron microscopy and diffractometry of materials, Springer, Berlin, 3rd edn, 2008.

45 J. Gallo, M. Slouf and S. B. Goodman, J. Biomed. Mater. Res., Part B, 2010, 94B(1), 171-177.

46 M. Slouf, A. Ostafinska, M. Nevoralova and I. Fortelny, Polym. Test., 2015, 42, 8-16.

47 P. Fulín, D. Pokorný, M. Slouf, M. Lapčíková, E. Pavlova and E. Zolotarevová, Acta Chir. Orthop. Traumatol. Cech., 2011, 78(2), 131-137. 